\title{
Experiments and Numerical Simulations of Electrodynamic Tether
}

\author{
By Kentaro IKI ${ }^{1)}$, Satomi KAWAMOTO ${ }^{2)}$, Ayaka TAKAHASHI ${ }^{3)}$, Tomori ISHIMOTO ${ }^{4)}$, \\ Atsushi YANAGIDA ${ }^{1)}$ and Susumu TODA ${ }^{1)}$ \\ ${ }^{1)}$ Waseda University, Tokyo, Japan \\ ${ }^{2)}$ Japan Aerospace Exploration Agency (JAXA), Tokyo, Japan \\ ${ }^{3)}$ Advanced Institute of Industrial Technology, Tokyo, Japan \\ ${ }^{4)}$ Kogakuin University, Tokyo, Japan
}

(Received June 17th, 2011)

\begin{abstract}
As an effective means of suppressing space debris growth, the Aerospace Research and Development Directorate of the Japan Aerospace Exploration Agency (JAXA) has been investigating an active space debris removal system that employs highly efficient electrodynamic tether (EDT) technology for orbital transfer. This study investigates tether deployment dynamics by means of on-ground experiments and numerical simulations of an electrodynamic tether system. Some key parameters used in the numerical simulations, such as the elastic modulus and damping ratio of the tether, the spring constant of the coiling of the tether, and deployment friction, must be estimated, and various experiments are conducted to determine these values. As a result, the following values were obtained: The elastic modulus of the tether was $40 \mathrm{GPa}$, and the damping ratio of the tether was 0.02 . The spring constant and the damping ratio of the tether coiling were $10^{-4} \mathrm{~N} / \mathrm{m}$ and 0.025 respectively. The deployment friction was $0.038 v+0.005 \mathrm{~N}$. In numerical simulations using a multiple mass tether model, tethers with lengths of several kilometers are deployed and the attitude dynamics of satellites attached to the end of the tether and tether libration are calculated. As a result, the simulations confirmed successful deployment of the tether with a length of $500 \mathrm{~m}$ using the electrodynamic tether system.
\end{abstract}

Key Words: Electrodynamic Tether, Experiments, Numerical Simulations, Deployment

\section{Introduction}

The amount of space debris is ever increasing, and its growth has been particular rapid in recent years. Consequently, pollution of the space environment has become a serious problem that can no longer be ignored. Space debris poses a serious collision risk to operating space systems, and requires the design of protection to survive collisions with small debris particles and maneuvers to avoid larger pieces. Collision avoidance maneuver is a particularly heavy burden on spacecraft designers and operators. Moreover, a huge quantity of smaller debris could be generated by collisions between larger objects. Some studies indicate that catastrophic collisions will occur every five to ten years mainly between 700 and $1000 \mathrm{~km}$ altitudes, and so the number of debris objects will increase even with no new launches ${ }^{1,2)}$. Therefore, the active removal of large space debris from crowded economically useful orbits (800-1500 km alt.) should begin as soon as possible.

The Japan Aerospace Exploration Agency (JAXA) has been investigating an active space debris removal system that captures large debris objects in crowded orbits to dispose of them. However, it requires a high cost to deorbit large debris objects from crowded regions using conventional propulsion because a large mass of propellant is required. From this perspective, electrodynamic tether (EDT) systems are very promising since they can generate sufficient thrust for orbital transfers without the need for propellant by utilizing interactions with Earth's magnetic field. Previous studies have shown that an EDT could deorbit many large debris items from the crowded orbits within one year ${ }^{3,4}$. Furthermore, the thrust of the EDT is so low that it does not have to be as firmly attached to the target as a conventional propulsion system, so attaching it to a debris object by a robot arm will be less challenging. A flight demonstration using a small satellite is being planned as a first step towards realizing an EDT debris removal system.

An electrodynamic tether with a length of several kilometers should be deployed on the orbits. Some flight demonstrations of tether deployment have been conducted, but many system structures have been complicated and large. An EDT debris removal system requires simple and compact structures of its components. From this viewpoint, we adopt a net-type bare tether and a spool-type reel deployed from the inside as simple and compact components of an EDT debris removal system. We have been conducting various ground-based experiments of the tether deployment using these components.

This study investigates tether deployment dynamics by means of numerical simulations of an EDT debris removal system. Many investigators have ever studied tether simulations. In many of these simulations, however, models were plain and experimentally-based parameters were not used. In this study, detailed models and experimentally-based parameters are used in the numerical simulations. Therefore, this investigation is meaningful for tether deployment of EDT debris removal systems. 
In this study, some key parameters used in the numerical simulations, such as the elastic modulus and damping ratio of the tether, the spring constant and damping ratio of the coiling of the tether, and deployment friction, must first be estimated by various ground-based experiments. Tether deployment is then analyzed by numerical simulation using the values determined by the experiments.

\section{EDT System}

\subsection{Principle of EDT system}

The principle of the EDT system is shown in Fig. 1. An electrodynamic tether is deployed by ejecting an end-mass from a small satellite. An electromotive force is generated as the conductive tether moves through the geomagnetic field in its orbit around the Earth. If a pair of plasma contactors at either end of the tether emits and collects electrons, the circuit is closed via the ambient plasma and an electric current flow through the tether. Interaction between the current and the geomagnetic field then generates a Lorentz force on the tether which acts opposite to the direction of flight. The EDT system can, therefore, provide deceleration without propellant, and shows promise as a high efficiency propulsion system for deorbiting space debris.

Many tether flight experiments have been conducted, but tether deployment has ended in failure in some of the experiments. Therefore, the crucial tether deployment dynamics needs to be analyzed to ensure a successful tether flight experiment.

\subsection{Tether and reel type}

A flight demonstration using a small satellite is planned to validate the EDT system. The tether and reel type used in the flight experiment are described below.

A net-type bare tether is adopted as the electrodynamic tether (Fig. 2). Since a bare tether can collect electrons directly from the ambient plasma, it is simpler and lighter than using other devices to collect electrons. A net-type tether can survive collisions with small debris and micrometeoroids because it has a multiple yarns that can maintain the tether's function even if one yarn is severed by a small debris particle or a micrometeoroid. Since the tether is several kilometers long, it is wound on a reel for launch and then deployed in space. However, it is possible that friction between parts of the bare tether in mutual contact might be great enough to stop deployment. Furthermore, the tether will become coiled when it is deployed from the reel (Fig. 3), and the effect of this coiling must be taken into consideration.

It is important to choose a reel with low deployment friction, and with this consideration a spool-type reel deployed from the inside, shown in Fig. 4 (C), is adopted. The reel is placed inside the end-mass and the tether is deploying by ejecting the end-mass from the satellite. The tether deployment is stopped by a braking mechanism at the final stage.

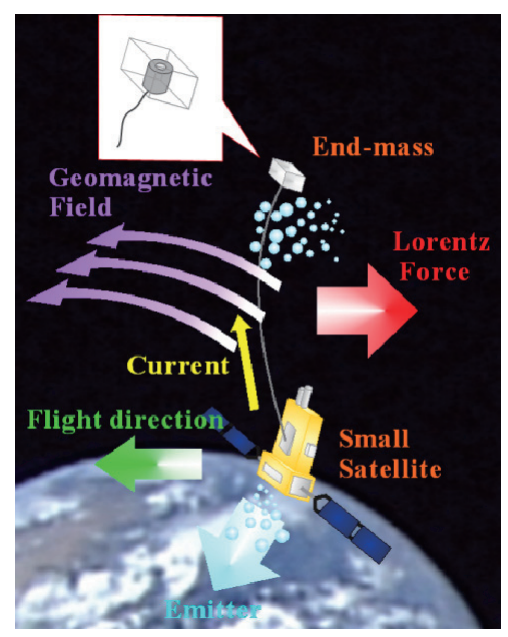

Fig. 1. Principle of EDT system.

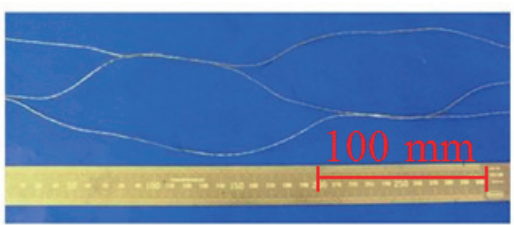

Fig. 2. Net-type bare tether composed of three yarns. Each yarn is made of aluminum and stainless steel wires. In this figure, the tether is extended in order to show the structure of net.

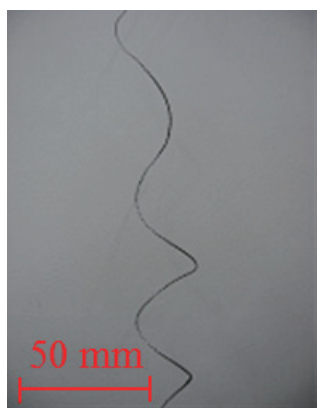

Fig. 3. Coiling of the tether.

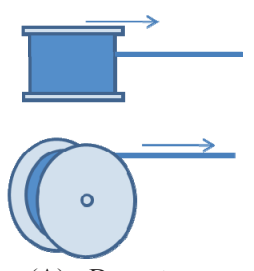

(A) Drum-type reel

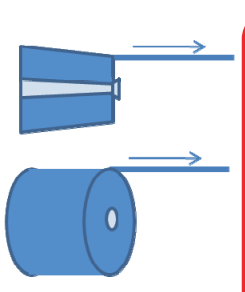

(B) Spool-type reel deployed from the outside

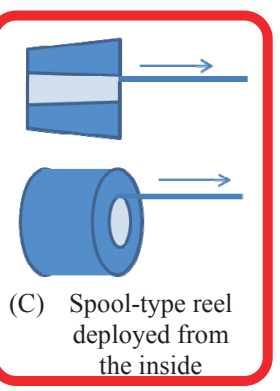

Fig. 4. Tether reel types.

\section{Experiments to Determine Parameter Values}

The values of some key parameters in the numerical simulations are estimated by experiments. The tether is modeled in the simulations as a lumped mass, that is, a chain of point masses connected to each other by a spring and viscous damper. The elastic modulus and damping ratio of the tether must be determined by measuring the properties of the 
physical tether. It is considered that the coiling of the tether may have an influence when the tether tension is low, such as during deployment. Accordingly, the spring constant and damping ratio of the coiling must also be estimated. Furthermore, deployment friction also plays a very important role during deployment and must be estimated. The experiments described in the following subsections were conducted to determine the values of these parameters.

\subsection{Tensile tests}

Tensile tests were conducted on a $200 \mathrm{~mm}$-long tether in order to estimate the elastic modulus of the tether from a stress-strain diagram. The result of the test is shown in Fig. 5. The estimated elastic modulus is about $40 \mathrm{GPa}$.

\subsection{Oscillation experiments to determine damping ratio}

Several types of tether were suspended vertically with a $1 \mathrm{~kg}$ mass attached to the end and their oscillations measured by an acceleration sensor. Since the measurements contained noise, the raw data were approximated by a following equation using the least-squares method (Powell method).

$$
y=A \exp (B t) \cos (C t+D)
$$

Where $A, B, C$ and $D$ are coefficients, and $t$ is time. Then, the damping ratio is expressed in the following equation by $B$ in Eq. (1), the weight of mass and the spring constant of the tether.

$$
\varsigma=-B \sqrt{\frac{m}{k}}
$$

Damping ratios are calculated from Eq. (2). An example of raw and processed tether oscillation damping curves obtained from this measurement is shown in Fig. 6. The function $A^{*} \exp \left(B^{*} t\right)$ indicates the envelope curve.

The damping ratios of the various tethers are shown in Fig. 7. The damping ratio of an $\mathrm{Al}$ net-type tether is about 0.02 . It was found that the damping ratio depends on the constituent material but is independent on the tether length.

Furthermore, numerical simulations of a spring-damper system were conducted in order to investigate the influence of atmospheric drag. The equation of the motion is shown in Eq. (3).

$$
\begin{gathered}
m \ddot{x}+c \dot{x}+k x+m g+d r a g=0 \\
d r a g=\frac{1}{2} C_{D} \rho S \dot{x}^{2}
\end{gathered}
$$

Where $m, c, k, \operatorname{drag}, C_{D}, \rho$, and $S$ are the mass of the attached mass, the damping ratio, the spring constant, the atmospheric drag, the coefficient of drag, the atmospheric density, and the reference area of the attached mass and tether, respectively.

It is found that damping by atmospheric drag is much smaller than damping by tether itself ${ }^{6}$. The influence of the atmospheric drag is therefore considered to be negligible.

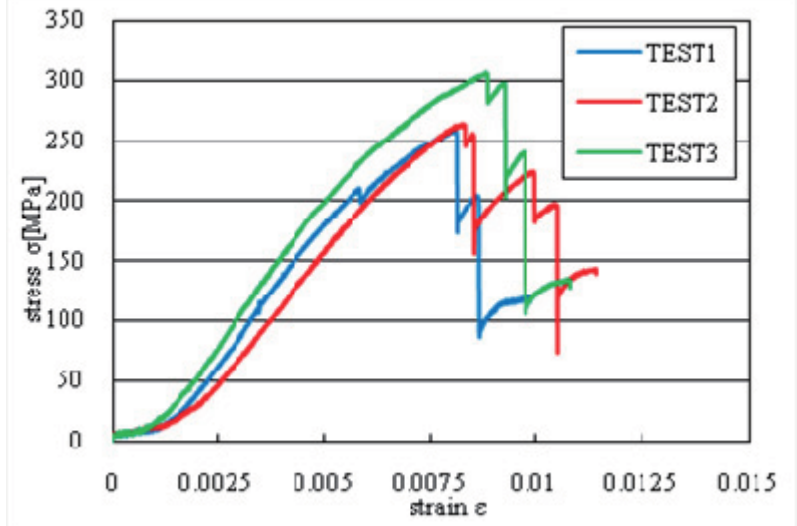

Fig. 5. Stress-strain diagram. 3 tests were conducted repeatedly under the same conditions. TESTs 1-3 are the cases of $200 \mathrm{~mm}$-long tether composed of stainless steel and aluminum wires.

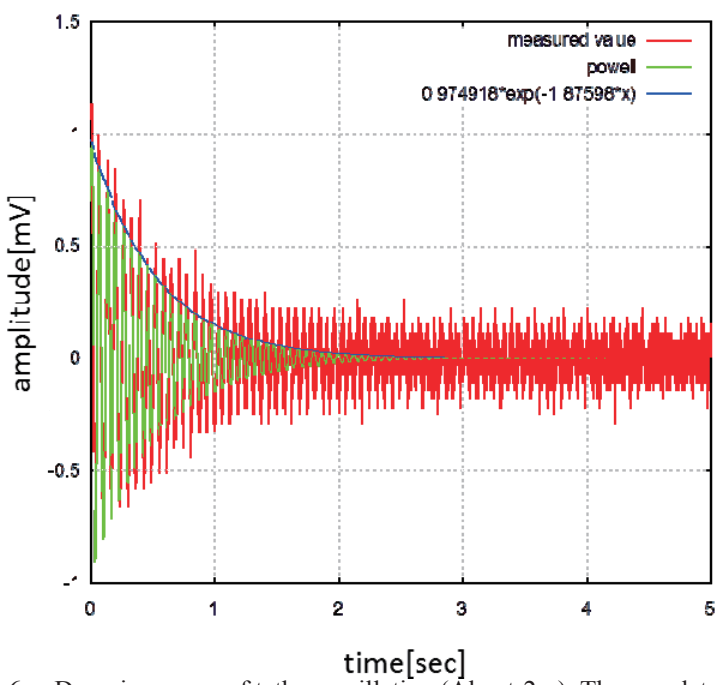

Fig. 6. Damping curve of tether oscillation (Al net-2m). The raw data were approximated by Powell method for noise removal.

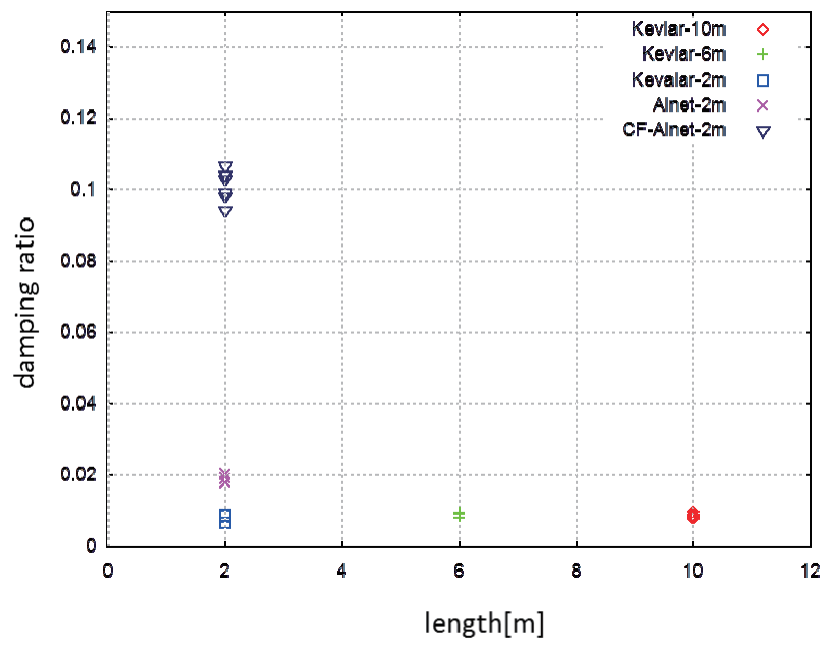

Fig. 7. Damping ratios of tethers of different materials. This figure shows the results of the oscillation experiments to determine damping ratio. The damping ratio of an $\mathrm{Al}$ net-type tether is about 0.02 . 


\subsection{Oscillation experiments to measure coiling}

Oscillation experiments were conducted in order to measure the spring constant and the damping ratio of the coiling of the tether. The experimental setup is shown in Fig. 8. The tether is suspended with a mass on the end, with the mass is light enough to keep the coiling. The oscillation frequency of the coiling is measured using stereo video cameras.

As an example, the motion of a mass attached to a $120 \mathrm{~cm}$-long Al net-type tether is shown in Fig. 9. Table. 1 shows the measured spring constant of the coiling. For this type of tether, the value is about $10^{-4} \mathrm{~N} / \mathrm{m}$ per $50 \mathrm{~m}$ of tether.

The damping curve was calculated from the time history of mass position using the least-squares method. Fig. 10 shows the damping ratios for various values of attached mass. In this study, the estimated damping ratio is about 0.025 . In addition, the influence of atmospheric drag was investigated by numerical simulations of a spring-damper system. The equation of the motion is shown in Eq. (4).

$$
\begin{gathered}
m \ddot{x}+c \dot{x}+k x+m g+d r a g=0 \\
d r a g=\frac{1}{2} C_{D} \rho S \dot{x}^{2}
\end{gathered}
$$

Where $m, c, k$,drag, $C_{D}, \rho$, and $S$ are the mass of the attached mass, the damping ratio of the coiling, the spring constant of the coiling, the atmospheric drag, the coefficient of drag, the atmospheric density, and the reference area of the attached mass and tether, respectively.

It is found that atmospheric drag increases damping ration by only a few percent ${ }^{5)}$. The influence of the atmospheric drag is therefore considered to be negligible.

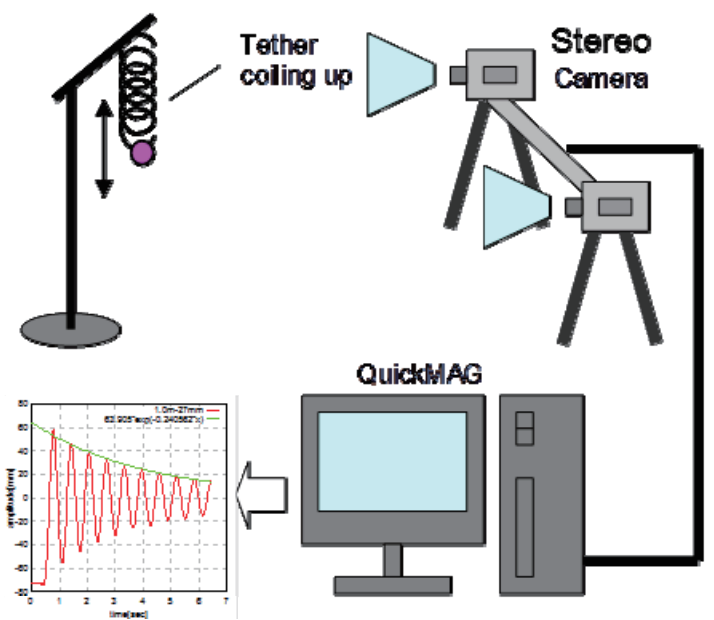

Fig. 8. Experimental setup of the oscillation experiments to measure coiling. "QuickMAG" is a three-dimensional measuring device.

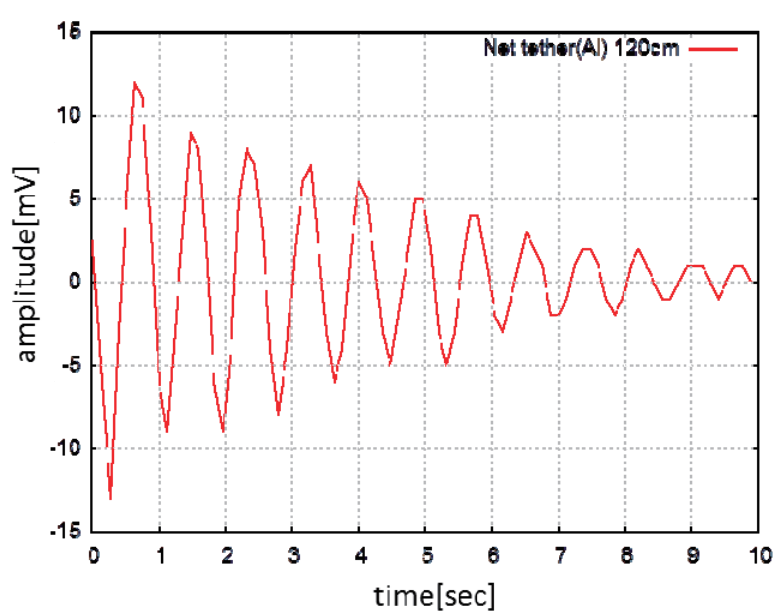

Fig. 9. Damping curve by oscillation of the coiling Al net-type tether.

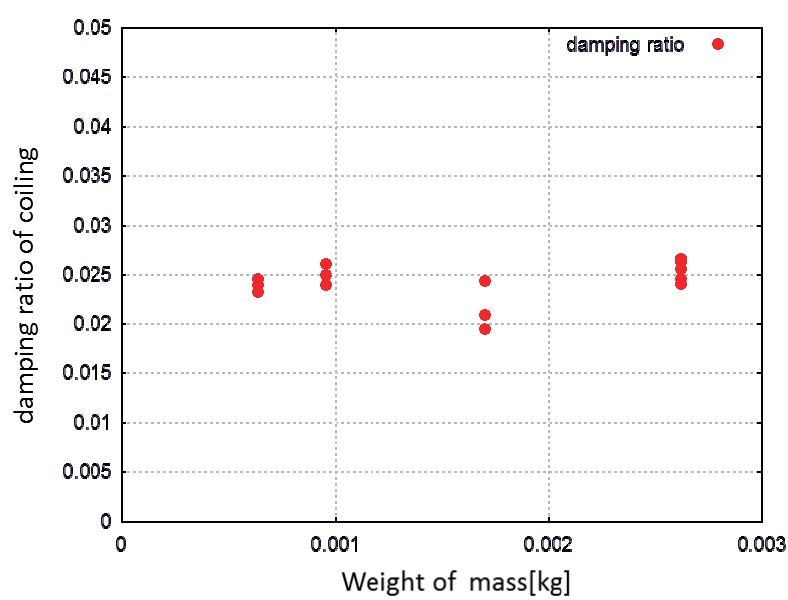

Fig. 10. Estimated damping ratio. I ne weignt or mass attached to the end of the tether is varied (Fig. 8).

Table. 1. Results of analysis of the spring constant of coiling.

\begin{tabular}{|c|c|c|c|}
\hline Tether type & $\begin{array}{c}\text { Length } \\
\mathrm{cm}\end{array}$ & $\begin{array}{c}\text { Frequency } \\
\mathrm{Hz}\end{array}$ & $\begin{array}{c}\text { Spring constant } \\
\text { per 50m N/m }\end{array}$ \\
\hline \multirow{3}{*}{$\begin{array}{c}\text { Al net-type } \\
\text { tether }\end{array}$} & 60 & 1.67 & $6.58 \mathrm{E}-05$ \\
\cline { 2 - 4 } & 90 & 1.61 & $9.24 \mathrm{E}-05$ \\
\cline { 2 - 4 } & 120 & 1.43 & $9.67 \mathrm{E}-05$ \\
\cline { 2 - 4 } & 180 & 1.28 & $9.73 \mathrm{E}-05$ \\
\hline \multirow{2}{*}{$\begin{array}{c}\text { Al and C } \\
\text { net-type } \\
\text { tether }\end{array}$} & 120 & 1.34 & $1.28 \mathrm{E}-04$ \\
\hline
\end{tabular}

\subsection{Tether deployment test on the ground}

EDT deployment using a net-type bare tether and spool-type reel deployed from inside was demonstrated. Here, the deployment friction of this mechanism was estimated.

The experiment setup is shown in Fig. 11. An end-mass carrying the tether and reel inside floats on an air table. The tether is deployed by ejecting the end-mass from a fixed base representing a small satellite using a spring (Fig. 12). The acceleration is estimated using the least-squares method (Powell method) from the motion of the end-mass measured by $3 \mathrm{D}$ stereo cameras, and the deployment friction is 
calculated from the acceleration.

To eliminate the effect of the atmospheric drag, tests were conducted with and without the tether between the end-mass and small satellite to be compared. In this way, the influence of tether could be isolated.

According to past investigations ${ }^{8,9)}$, deployment friction varies with deployment velocity. Fig. 13 shows the measured relationship between acceleration and deployment velocity. It was attempted to fit a linear function to the data, but it proved difficult to estimate the constant term of the acceleration because it was a small value. The velocity-dependent term (the slope of the function) was, therefore, estimated here, and the constant term was measured in a separate experiment. The result of velocity-dependent term is shown in Table. 2 .

Next, the constant term was measured. As shown in Fig. 14, an external force was applied to an end-mass at rest. The constant term can be estimated by measuring the applied force at which the end-mass starts to move. The values measured in this experiment were in the range $0-0.005 \mathrm{~N}$. In this investigation, $0.005 \mathrm{~N}$, which is greater than the true value, is adopted as the constant term of deployment friction for use in the numerical simulations.

From the above experiments, the deployment friction $F_{\text {deploy }}$ was estimated as follows:

$$
F_{\text {deploy }}=0.038 v+0.005 \quad[\mathrm{~N}]
$$

This experimental value of deployment friction seems to be greater than the true value because each term was determined separately. However, if numerical simulations confirm the feasibility of tether deployment with this experimental value, it is expected that the EDT system in the demonstration flight will also be able to deploy the full length of the tether because the numerical prediction should be conservative.

Fig. 15 shows the directions in which the end-mass was ejected. The variance is less than $0.02 \mathrm{rad}$. This confirms that this ejection mechanism can eject the end-mass in almost a straight direction.

\subsection{Other experiments}

Other experiments were also conducted to prove the EDT system. The bare tether collects electrons from the ambient plasma so that a current flows within the tether. Bias experiments of the bare tether in a vacuum chamber were conducted in order to investigate the interactions between the bare tether and ionospheric plasma ${ }^{10)}$. In a positive-bias experiment, electron current collection was confirmed and the conditions of anomalous current collection were investigated. In a negative-bias experiment, it was confirmed that negative arcs did not occur in the net-type bare tether proposed for the flight demonstration.

Collision of the tether with small debris objects and micrometeoroids is a serious issue that will limit the tether's lifetime. Hypervelocity impact tests using a two-stage light gas gun were conducted in order to investigate the mechanism of severing of the tether by small particles and to estimate the tether's lifetime. ${ }^{11)}$ It was found that a twisted tether made of aluminum and stainless steel wire has better survivability against hypervelocity impacts than a conventional non-conductive tether.

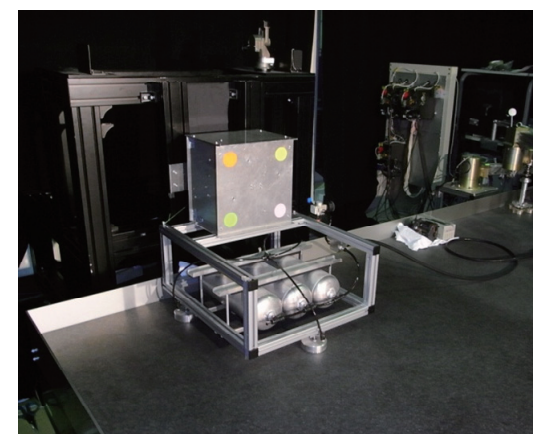

Fig. 11. Experiment apparatus modeling end-mass.

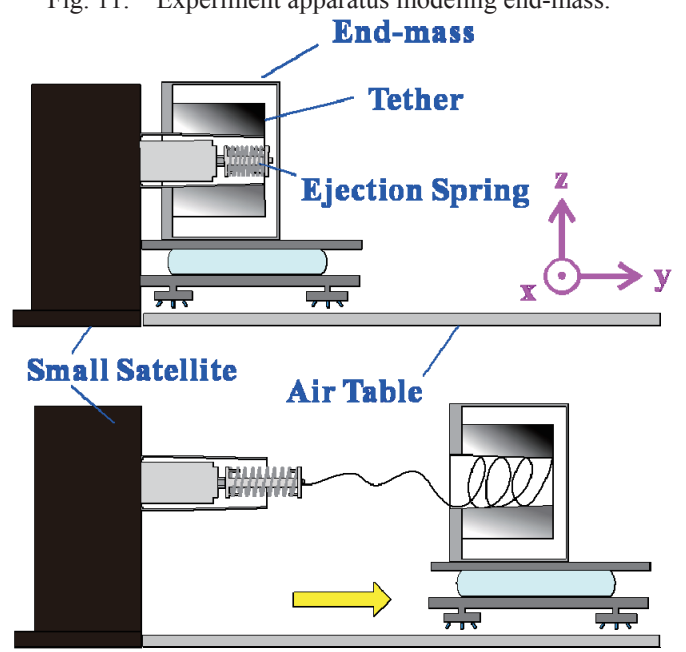

Fig. 12. Tether deployment test on the ground. The end-mass is ejected from the small satellite by a coil spring and the tether placed in the end-mass is deployed.

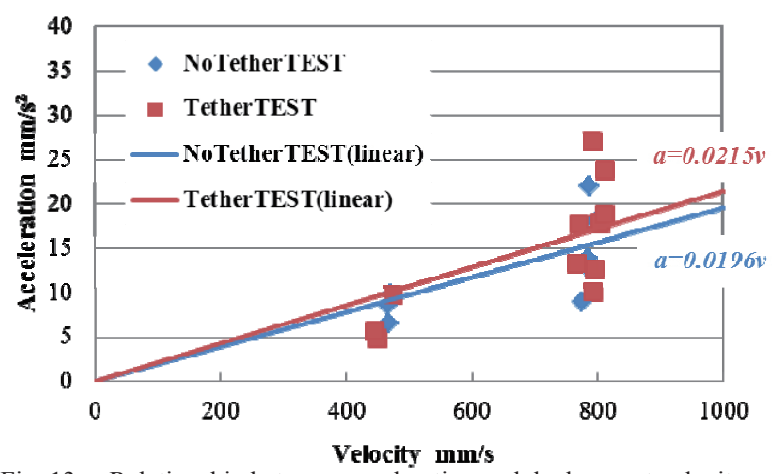

Fig. 13. Relationship between acceleration and deployment velocity.

\section{End-mass}

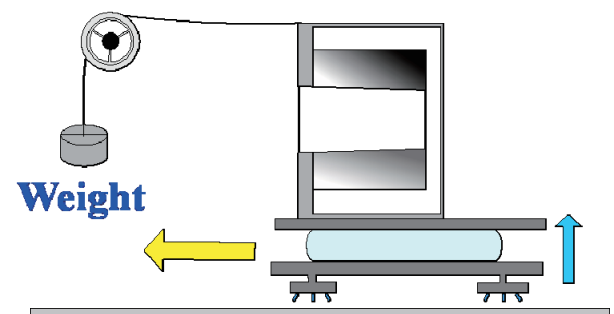

Fig. 14. Experiment measuring constant term of deployment friction. 


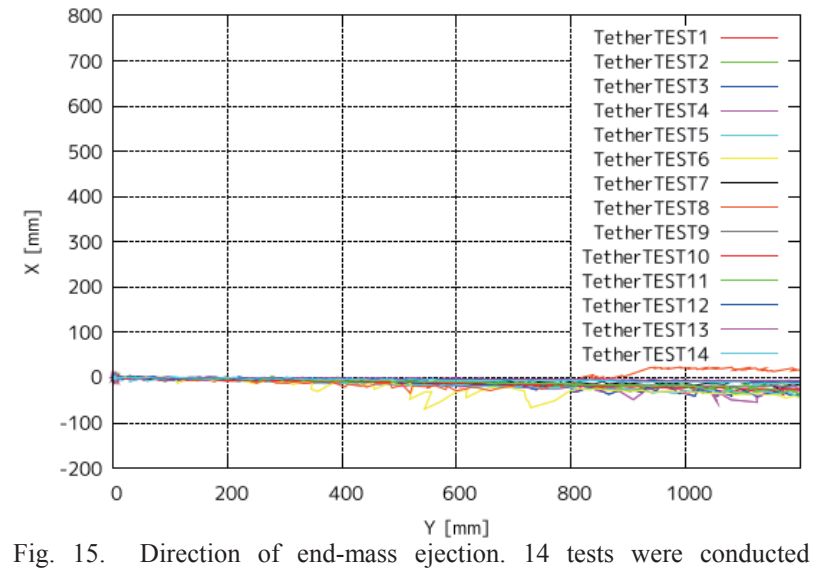
repeatedly under the same conditions. The variance is less than $0.02 \mathrm{rad}$.

Table. 2. Result of velocity-dependent term.

\begin{tabular}{|c|c|c|}
\hline \multirow{2}{*}{ Acceleration } & Without tether & $0.0196 v$ \\
\cline { 2 - 3 } & With tether & $0.0215 v$ \\
\cline { 2 - 3 } & Deployment & $0.0019 v$ \\
\hline $\begin{array}{l}\text { Deployment } \\
\text { friction N }\end{array}$ & $\begin{array}{c}\text { End-mass } \\
(20 \mathrm{~kg})\end{array}$ & $0.038 v$ \\
\hline
\end{tabular}

\section{Numerical Simulation of EDT System}

Tether deployment tests on the ground are not enough to confirm the dynamics of tether deployment on orbit, because the length that can be deployed in on-ground tests is too short and orbital motion effects such as the gravity gradient and Coriolis forces cannot be examined. The deployment dynamics on orbit are, therefore, investigated by numerical simulation.

\subsection{Model and conditions}

Fig. 16 shows an overview of the simulation model. To take into account tether flexibility, the tether is modeled as a lumped mass by dividing it into point masses connected to its neighbors by a spring and viscous damper. The equation of motion of each point mass is formulated in a coordinate system in which the origin is the center of mass of the system rotating around the Earth (Fig. 17). The deployment of the tether is modeled by adding point masses to the lumped mass model (Fig. 18). The equations of motion of the $i$-th point mass in this rotating coordinate system are as follows:

$$
\begin{aligned}
\ddot{x}_{i}= & \left(r+y_{i}\right) \ddot{\theta}+2\left(\dot{r}+\dot{y}_{i}\right) \dot{\theta}+x_{i} \dot{\theta}^{2}-\mu_{e} x_{i}\left[x_{i}^{2}+\left(y_{i}+r\right)^{2}+z_{i}^{2}\right]^{-\frac{3}{2}} \\
& -\frac{\alpha_{i} k_{i}}{m_{i}}\left(x_{i}-x_{i+1}\right)+\frac{\alpha_{i-1} k_{i-1}}{m_{i}}\left(x_{i-1}-x_{i}\right)-\frac{c_{i}}{m_{i}}\left(\dot{x}_{i}-\dot{x}_{i+1}\right)+\frac{c_{i-1}}{m_{i}}\left(\dot{x}_{i-1}-\dot{x}_{i}\right)+\frac{Q_{x i}}{m_{i}} \\
\ddot{y}_{i}= & -\ddot{r}-x_{i} \ddot{\theta}-2 \dot{\dot{x}_{i}} \dot{\theta}+\left(r+y_{i} \dot{\theta}^{2}-\mu_{e}\left(y_{i}+r\right)\left[x_{i}^{2}+\left(y_{i}+r\right)^{2}+z_{i}^{2}\right]^{\frac{3}{2}}\right. \\
& -\frac{\alpha_{i} k_{i}}{m_{i}}\left(y_{i}-y_{i+1}\right)+\frac{\alpha_{i-1} k_{i-1}}{m_{i}}\left(y_{i-1}-y_{i}\right)-\frac{c_{i}}{m_{i}}\left(\dot{y}_{i}-\dot{y}_{i+1}\right)+\frac{c_{i-1}}{m_{i}}\left(\dot{y}_{i-1}-\dot{y}_{i}\right)+\frac{Q_{y i}}{m_{i}} \\
\ddot{z}_{i}= & -\mu_{e} z_{i}\left[x_{i}^{2}+\left(y_{i}+r\right)^{2}+z_{i}^{2}\right]^{\frac{3}{2}}-\frac{\alpha_{i} k_{i}}{m_{i}}\left(z_{i}-z_{i+1}\right)+\frac{\alpha_{i-1} k_{i-1}}{m_{i}}\left(z_{i-1}-z_{i}\right) \\
& -\frac{c_{i}}{m_{i}}\left(\dot{z}_{i}-\dot{z}_{i+1}\right)+\frac{c_{i-1}}{m_{i}}\left(\dot{z}_{i-1}-\dot{z}_{i}\right)+\frac{Q_{z i}}{m_{i}} \\
\alpha_{i}= & 1-\frac{l_{0}}{\sqrt{\left(x_{i}-x_{i+1}\right)^{2}+\left(y_{i}-y_{i+1}\right)^{2}+\left(z_{i}-z_{i+1}\right)^{2}}}
\end{aligned}
$$

where $r, \theta, \mu_{e}, c, k, m$, and $Q$ are, respectively, the radius of the orbit, the orbital angular velocity of the center of mass of the tether system, the gravitational constant of the Earth, the damping factor of the tether, the spring constant of the tether, the mass of each point mass and the total force acting on it. The IGRF 2000 (International Geomagnetic Reference Field) (10x10) and EGM 96 (Earth Gravitational Model) (10x10) models are used for the geomagnetic field and the Earth's geo-potential field, respectively. The principal parameters used in the numerical simulations are shown in Table. 3.

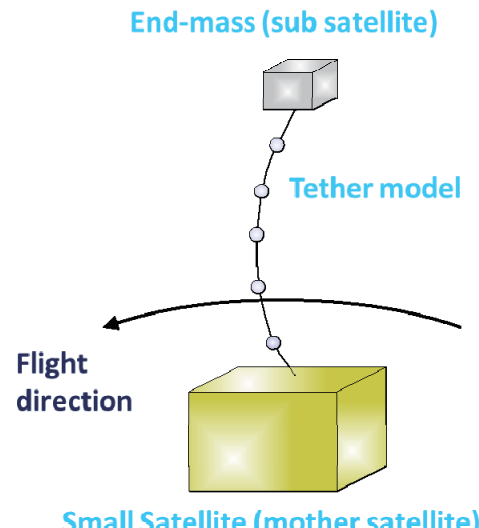

Fig. 16. Simulation model overview.

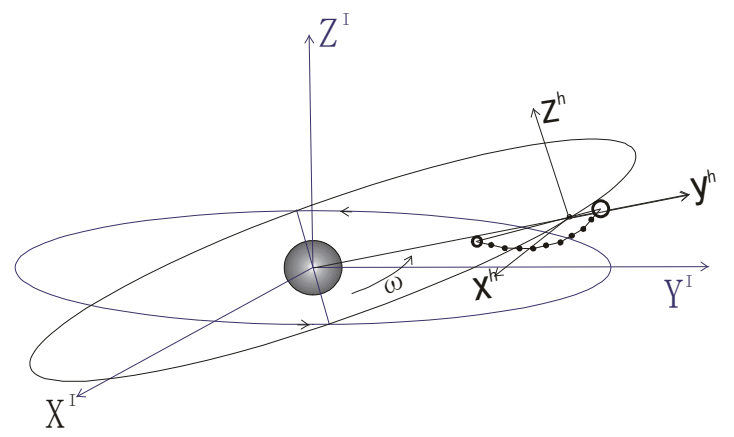

Fig. 17. Rotating coordinate system around the Earth $\left(\mathrm{X}^{\mathrm{h}}, \mathrm{Y}^{\mathrm{h}}, \mathrm{Z}^{\mathrm{h}}\right)$.

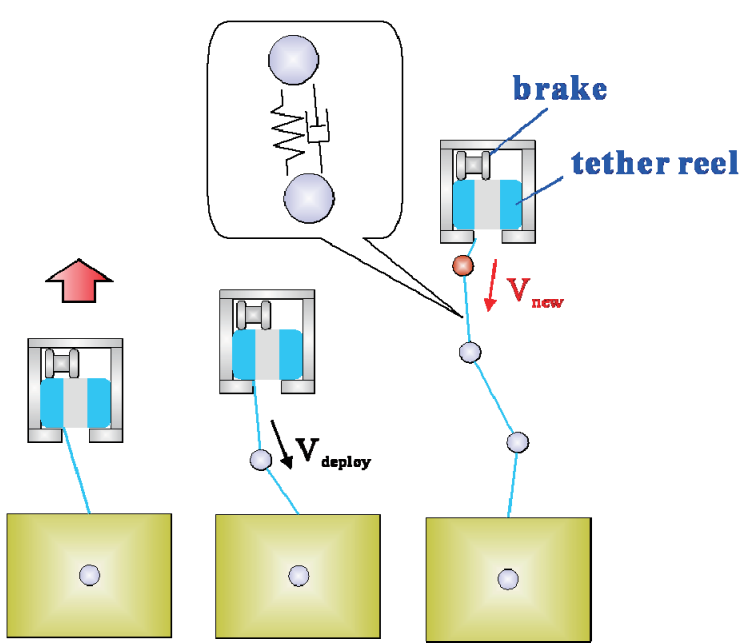

Fig. 18. Tether deployment model. The reel and the brake are placed in the end-mass during deployment. The brake model operates at the end of the deployment phase. 
Table. 3. Simulation conditions.

\begin{tabular}{|c|c|c|c|}
\hline \multicolumn{2}{|l|}{ Calculation time } & $\sec$ & 20000.0 \\
\hline \multicolumn{2}{|l|}{ Time step } & $\sec$ & 0.00005 \\
\hline \multirow{3}{*}{ Orbit } & long radius & $\mathrm{km}$ & 7428 \\
\hline & eccentricity & & 0.02019 \\
\hline & orbital inclination & deg & 31 \\
\hline $\begin{array}{c}\text { Small satellite } \\
\text { (without } \\
\text { end-mass) }\end{array}$ & mass & $\mathrm{kg}$ & 349.5 \\
\hline \multirow{2}{*}{$\begin{array}{c}\text { End-mass } \\
\text { (containing tether) }\end{array}$} & mass & $\mathrm{kg}$ & 16 \\
\hline & initial velocity & $\mathrm{m} / \mathrm{s}$ & 1.0 \\
\hline \multirow{5}{*}{ Tether } & Length & $\mathrm{m}$ & 500 \\
\hline & point mass number & & 21 \\
\hline & line density & $\mathrm{kg} / \mathrm{m}$ & 0.001 \\
\hline & elastic modulus & $\mathrm{GPa}$ & 40 \\
\hline & damping ratio & & 0.02 \\
\hline \multirow{2}{*}{ Coiling of tether } & spring constant & $\mathrm{N} / \mathrm{m}$ & $1.0 \mathrm{e}-4$ \\
\hline & damping ratio & & 0.025 \\
\hline \multirow{2}{*}{$\begin{array}{l}\text { Deployment } \\
\text { friction }\end{array}$} & $\begin{array}{l}\text { velocity-dependent } \\
\text { term }\end{array}$ & $\mathrm{N}$ & $0.038 v$ \\
\hline & constant term & $\mathrm{N}$ & 0.005 \\
\hline
\end{tabular}

\subsection{Numerical simulations results}

Tether deployment using the EDT mechanism proposed for the flight demonstration was analyzed by numerical simulation. Fig. 19 shows the changes in the form of tether during deployment. The tether librates as it is swung opposite to the flight direction by Coriolis force at the beginning of deployment and then is swung in the former direction again by the gravity gradient force. The in-plane libration angle is less than $40 \mathrm{deg}$. From this simulation, the tether can be deployed out to $500 \mathrm{~m}$, the planned length.

In some previous tether flight experiments, tether deployment stopped halfway because the deployment friction was greater than expected. According to the past investigation $^{12)}$, the friction of metal surfaces in a vacuum is several times greater than in the atmosphere owing to the mutual adhesion of metal surfaces in vacuum conditions. In this study, the tether deployment friction in a vacuum is assumed to be 1.2-1.5 times greater than in the atmosphere because the macroscopic friction such as tangling of the tether, as well as the adhesion of metal surfaces, plays an important role in the tether deployment friction. To investigate the effects of greater than anticipated friction, cases with the value of friction set at 1.5 times and 2.0 times the nominal value $F_{\text {deploy }}$ determined from on-ground tests were also analyzed. As shown in Figs. 20 and 21, in both cases the friction is so great that deployment stops halfway. In the case of 1.5 times the nominal friction, deployment restarts and the tether deployed to $500 \mathrm{~m}$ since the gravity gradient force is increased by in-plane libration. In the case of 2.0 times, however, the tether cannot be deployed to its full length. It is estimated that the gravity gradient force is insufficient to restart deployment since the in-plane libration angle gradually decays (Fig. 22).

Furthermore, the effect of different initial end-mass ejection velocities should be analyzed in order to determine the optimum speed to eject the end-mass. As shown in Fig. 23, deployment stops immediately if the end-mass is ejected at $0.2 \mathrm{~m} / \mathrm{s}$. To deploy whole tether length, the initial velocity must be greater than $0.4 \mathrm{~m} / \mathrm{s}$. As shown in Fig. 24, deployment stops once before the brake is applied if the end-mass is ejected at under $0.6 \mathrm{~m} / \mathrm{s}$. To deploy the whole tether length without any stops, over $0.6 \mathrm{~m} / \mathrm{s}$ is required. Although deployment can be restarted by in-plane oscillation of the tether, $0.2 \mathrm{~m} / \mathrm{s}$ is such a low velocity that tether deployment cannot start again. Therefore, a sufficient initial velocity is required.

Next, the influence of different tether deployment lengths was analyzed. This study investigated cases of $500 \mathrm{~m}, 1000 \mathrm{~m}$ and $2000 \mathrm{~m}$. As shown in Fig. 25, $500 \mathrm{~m}, 1000 \mathrm{~m}$ and $2000 \mathrm{~m}$ tethers can be deployed full length. As shown in Fig. 26, the velocity initially decreases due to deployment friction and then increases again due to the gravity gradient. Since the longer tethers are subjected more to the gravity gradient, the velocity immediately before brake application is higher.

Normally, the small satellite will be controlled to maintain a constant attitude, but it is possible that the torque caused by tether tension might be beyond the capability of the satellite's attitude control. Accordingly, cases with and without attitude control were compared. (I, J, K) indicates the position vector of rigid body in the rotating coordinate system around the Earth namely the hill coordinate system (Fig. 27). Fig. 28 shows the time history of $\mathrm{Y}$ components of vector $\mathrm{K}$, which signifies the change of satellite attitude. For the uncontrolled case, the attitude oscillates with a short period, about $600 \mathrm{sec}$, and as shown in Fig. 29, the torque oscillates with the same period. However, there is no difference in tether deployment between the controlled and uncontrolled cases.

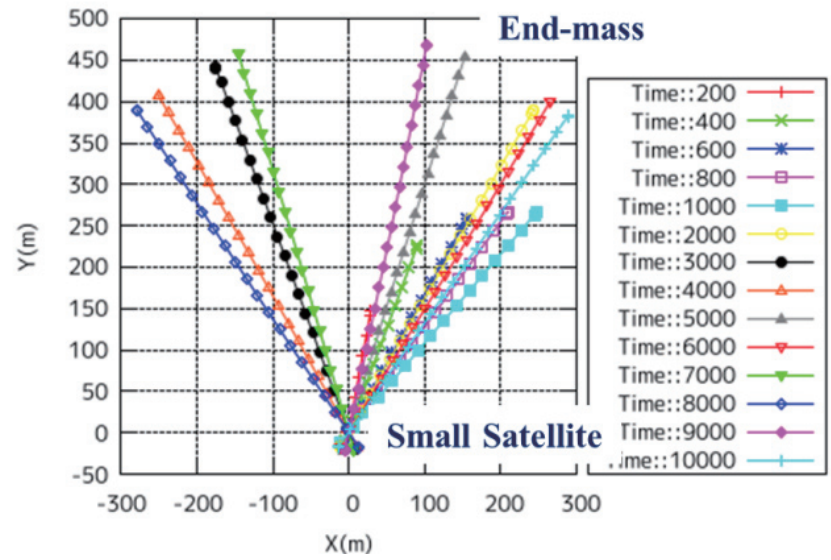

Fig. 19. Change in form of tether during deployment. The origin is the center of mass of the system (tether length: $500 \mathrm{~m}$, initial velocity: $1.0 \mathrm{~m} / \mathrm{s}$, $F_{\text {deploy }}=0.038 v+0.005 \mathrm{~N}$, with attitude control ). 


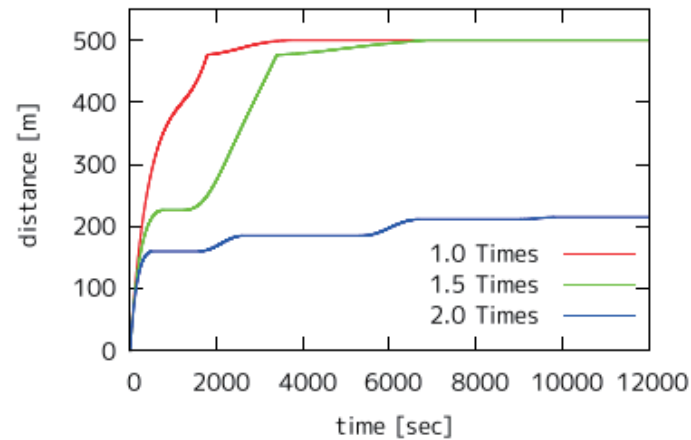

Fig. 20. Time history of distance between end-mass and satellite in the cases of various deployment frictions (tether length: $500 \mathrm{~m}$, initial velocity: $1.0 \mathrm{~m} / \mathrm{s}$, with attitude control). 1.0 times indicates $0.038 v+0.005 \mathrm{~N}$.

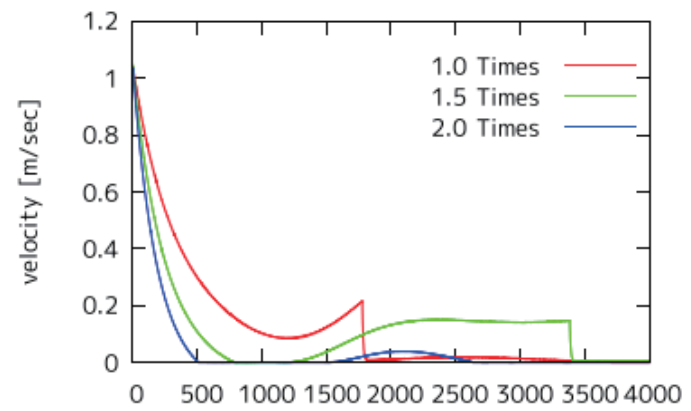

time $[\mathrm{sec}]$
Fig. 21. lime history of velocity in the cases of various deployment frictions (tether length: $500 \mathrm{~m}$, initial velocity: $1.0 \mathrm{~m} / \mathrm{s}$, with attitude control). 1.0 times indicates $0.038 v+0.005 \mathrm{~N}$.

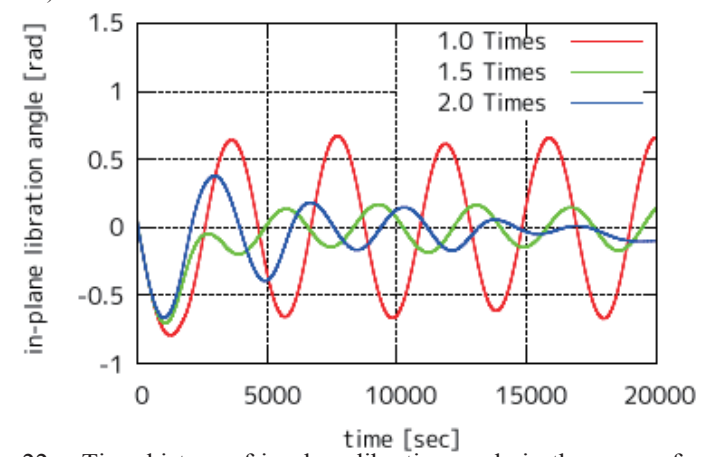

Fig. 22. Time history of in-plane libration angle in the cases of various deployment frictions (tether length: $500 \mathrm{~m}$, initial velocity: $1.0 \mathrm{~m} / \mathrm{s}$, with attitude control). 1.0 times indicates $0.038 v+0.005 \mathrm{~N}$.

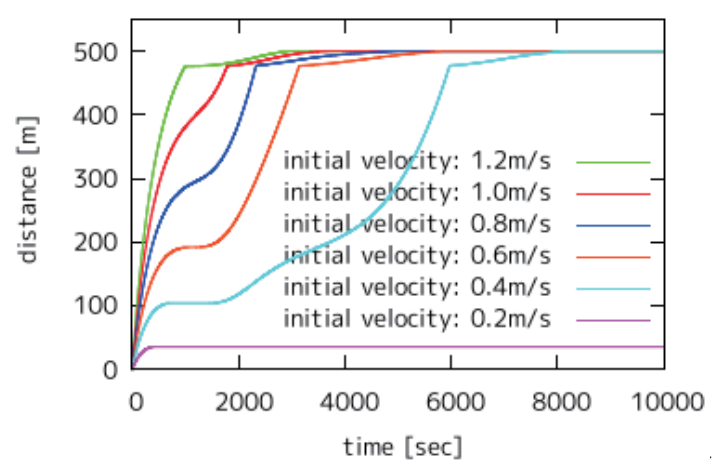

time [sec]
Fig. 23. I 1 me nistory ot distance between end-mass and satellite in the cases of various initial velocities (tether length: $500 \mathrm{~m}$, $F_{\text {deploy }}=0.038 v+0.005 \mathrm{~N}$, with attitude control).

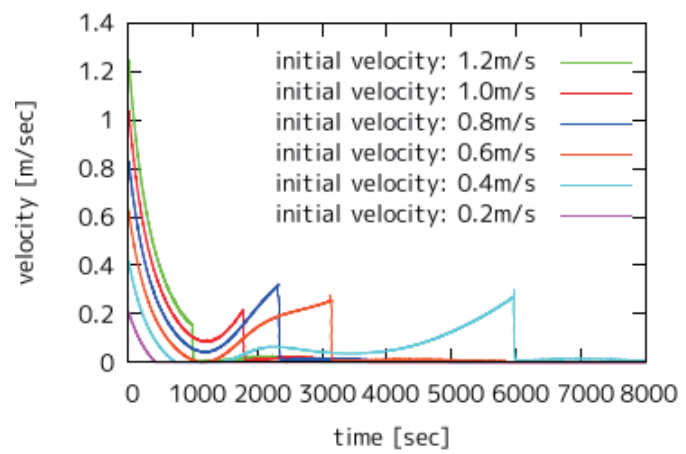

Fig. 24. Time history of velocity in the cases of various initial velocities (tether length: $500 \mathrm{~m}, F_{\text {deploy }}=0.038 v+0.005 \mathrm{~N}$, with attitude control).

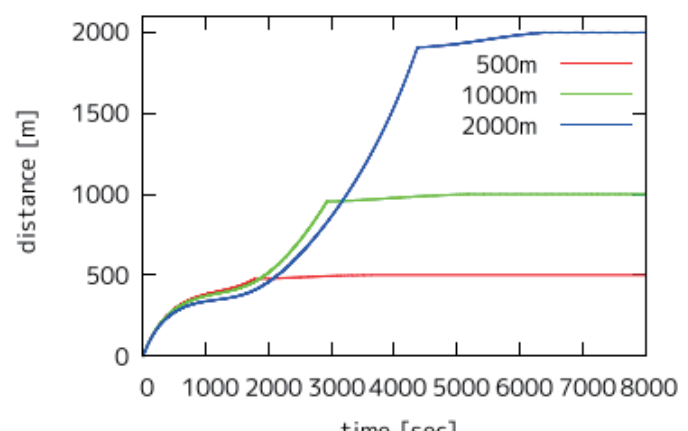

Fig. 25. Time history of distance between end-mass and satellite in the cases of various tether length (initial velocity: $1.0 \mathrm{~m} / \mathrm{s}$, $F_{\text {deploy }}=0.038 \mathrm{v}+0.005 \mathrm{~N}$, with attitude control).

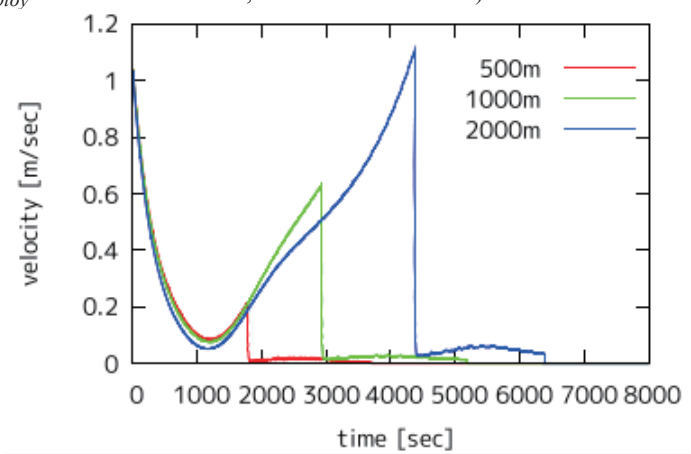

Fig. 26. Time history of velocity in the cases of various tether length (initial velocity: $1.0 \mathrm{~m} / \mathrm{s}, \quad F_{\text {deploy }}=0.038 v+0.005 \mathrm{~N}$, with attitude control).

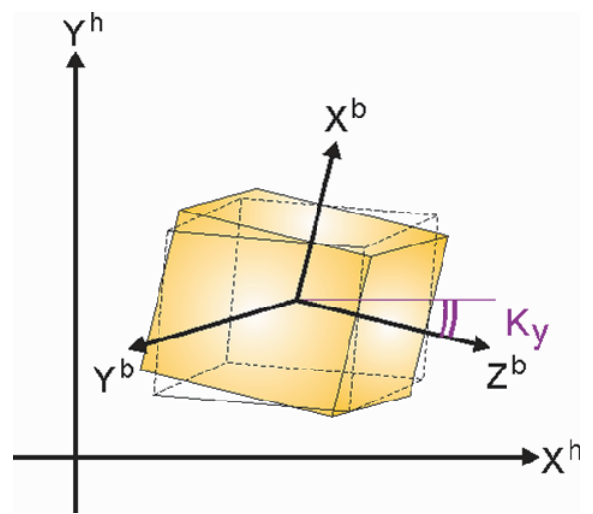

Fig. 27. Image of satellite attitude. (I, J, K) indicates the position vector of rigid body in the hill coordinate system. Ky is $\mathrm{Y}$ components of vector $\mathrm{K}$, which signifies the change of satellite attitude. 


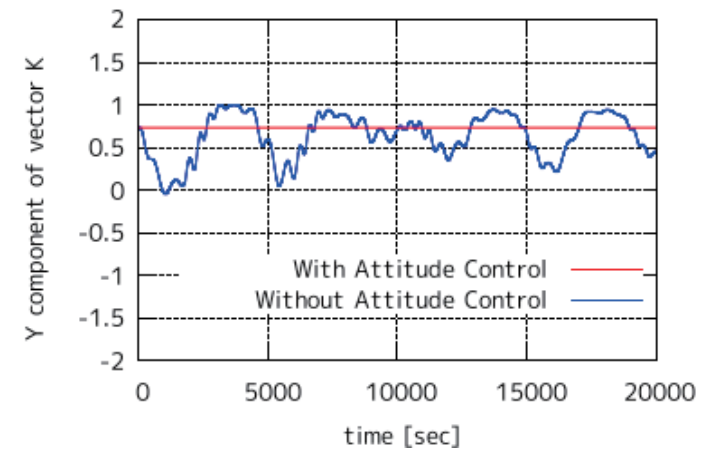

Fig. 28. Time history of attitude change in the cases with and without attitude control (tether length: $500 \mathrm{~m}$, initial velocity: $1.0 \mathrm{~m} / \mathrm{s}$, $F_{\text {deploy }}=0.038 v+0.005 \mathrm{~N}$ ). . This figure shows $\mathrm{Y}$ components of vector $\mathrm{K}$, which is $\mathrm{Z}$ component of body in the hill coordinate system.

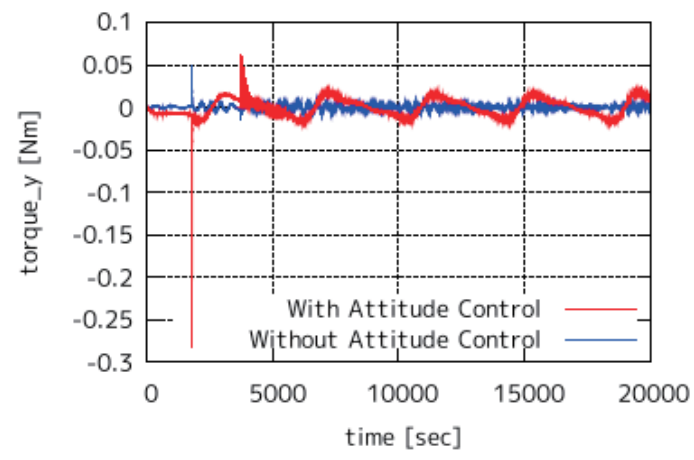

Fig. 29. Time history of torque in the cases with and without attitude control (tether length: $500 \mathrm{~m}$, initial velocity: $1.0 \mathrm{~m} / \mathrm{s}$, $\left.F_{\text {deploy }}=0.038 v+0.005 \mathrm{~N}\right)$.

\section{Conclusion}

This study investigated the tether deployment dynamics of an EDT debris removal system by numerical simulations. A net-type bare tether and a spool-type reel deployed from the inside are adopted as components of this EDT debris removal system because of these simple and compact structures. The parameters used in the numerical simulations were estimated from various experiments. As a result, the following values were obtained: The elastic modulus of the tether was $40 \mathrm{GPa}$, and the damping ratio of the tether was 0.02 . The spring constant and the damping ratio of the tether coiling were
$10^{-4} \mathrm{~N} / \mathrm{m}$ and 0.025 respectively. The deployment friction was $0.038 v+0.005 \mathrm{~N}$.

Then, the dynamics of tether deployment from a small satellite were analyzed by numerical simulations using these parameters. The simulations confirmed successful deployment of the tether with a length of $500 \mathrm{~m}$ using the EDT system proposed for the flight demonstration. In addition, the following influences were investigated: deployment friction in a vacuum, varying initial deployment velocity, different tether lengths, and difference between controlled and uncontrolled small satellite attitude.

\section{References}

1) Liou, J. $-\mathrm{C}$ and Johnson, N.: Risks in Space from Orbit Debris Science, 311(2006), pp.340-341.

2) Liou, J. $-\mathrm{C}$ and Johnson, N.: Instability of the present LEO satellite populations, Advances in Space Research 41(2008), pp. 1046-1053.

3) Kawamoto, S., Ohkawa, Y., et al.: Strategy for Active Debirs Removal Using an Electrodynamic Tether, ISTS-r-2-36, 2008.

4) Kawamoto, S., Makida, T., Sasaki, F. et al.: Precise Numerical Simulations of Electrodynamic Tethers for an Active Debris Removal System, IAC-05-C1.2.07.

5) Kobayashi, Y., Yamagiwa, Y., Otsu, H. and Kawamoto, S.: Deployment Dynamics of Electrodynamic Tether from a Small Satellite, AIAA $47^{\text {th }}$ Aerospace Science meeting, 2009.

6) Kawamoto, S., Umeoka, T. et al.: Gound Experiments to Asess the Deployment Dynamics of Electrodynamic Tethers, The $53^{\text {rd }}$ Space Science and Technology Conference, 1H19, 2009 (in Japanese).

7) Takahashi, A., Iki, K., Kawamoto, S.: Release and Deployment Experiments of Tethered satellite, IAC-10-D4.3.7.

8) Hoit, R.P. and Forward, R.L.: The Terminator Tether ${ }^{\mathrm{TM}}$ : Autonomous Deorbit of LEO Spacecraft for Space Debris Mitigation, $38^{\text {th }}$ Aerospace Sciences Meeting \& Exhibit, 2000.

9) Hoit, R.P, Forward, R.L. et. al: The RETRIEVE Microsatellite Tether Deorbit Experiment, Tethers Unlimited, Inc., 2002.

10) Koki Kashihara, Mengu Cho and Satomi Kawamoto: Ground Experiments and Computer Simulations of Interaction Between Bare Tether and Plasma, Plasma Science, IEEE Transactions on(2008), pp. 2324-2335.

11) Yanagida, A., Kawamoto, S., Higashide, M. et al.: Study of Hypervelocity Impact on Electrodynamic Tether for Tether Lifetime Evaluation, IAC-10.A6.3.8.

12) Shiomi, H. et al.: Friction and Wear Characteristics of Bare Tether, JAST Tribology Conference (Tokyo, 2010-5) (in Japanese). 